\title{
Performance Analysis of Compressive-Sensing-Based Through-the-Wall Imaging with Effect of Unknown Parameters
}

\author{
Muhammed Duman and Ali Cafer Gurbuz \\ Department of Electrical and Electronics Engineering, TOBB University of Economics and Technology, Sogutozu Caddesi No. 43, \\ 06560 Ankara, Turkey \\ Correspondence should be addressed to Ali Cafer Gurbuz, acgurbuz@etu.edu.tr
}

Received 4 March 2012; Accepted 1 May 2012

Academic Editor: Francesco Soldovieri

Copyright ( 2012 M. Duman and A. C. Gurbuz. This is an open access article distributed under the Creative Commons Attribution License, which permits unrestricted use, distribution, and reproduction in any medium, provided the original work is properly cited.

\begin{abstract}
Compressive sensing (CS) has been shown to be a useful tool for subsurface or through-the-wall imaging (TWI) using ground penetrating radar (GPR). It has been used to decrease both time/frequency or spatial measurements and generate high-resolution images. Although current works apply CS directly to TWI, questions on the required number of measurements for a sparsity level, measurement strategy to subsample in frequency and space, or imaging performance in varying noise levels and limits on CS range resolution performance still needs to be answered. In addition current CS-based imaging methods are based on two basic assumptions; targets are point like and positioned at only discrete grid locations and wall thickness and its dielectric constant are perfectly known. However, these assumptions are not usually valid in most TWI applications. This work extends the theory of CS-based radar imaging developed for subsurface imaging to TWI and outlines the performance of the proposed imaging for the above-mentioned questions using numerical simulations. The effect of unknown parameters on the imaging performance is analyzed, and it is observed that off-the-grid point targets and big modeling errors decreases the performance of CS imaging.
\end{abstract}

\section{Introduction}

Through-the-wall imaging (TWI) radar [1] has become an emerging technology in recent years due to its broad applications in both civilian and military sectors. In these applications, an electromagnetic wave, which could penetrate the wall, is transmitted and received at several points within a synthetic aperture to generate a vision of the scene. TWI applications generally require high-resolution images. High-range resolution requires use of an ultra-wideband (UWB) pulse, while high cross range resolution requires a very long aperture. A new technique for GPR and TWI resulting in high-resolution imaging using less number of measurements based on sparse recovery is the compressivesensing-based radar imaging [2-8].

Compressive sensing (CS) $[9,10]$ techniques enable reconstruction of unknown signals, which have a sparse representation in a certain transformation domain, from a set of measurements that can be significantly less than the number of unknowns. Since radar target scenes with strong reflectors and moving targets may admit a sparse representation, reconstruction of target scenes can be formulated as a sparse signal reconstruction problem. For a signal $x$ of dimension $N$, which has a $K$-sparse representation as $x=\Psi s$, CS takes $M=O(K \log N)$ nontraditional random linear measurements as $y=\Phi x$ and solves a convex $\ell_{1}$ minimization problem:

$$
\min \|s\|_{1}, \quad \text { subject to } y=\Phi \Psi s,
$$

to reconstruct the sparse signal $s$. In this way, instead of taking $N$ measurements, $M<N$ measurements is sufficient to reconstruct the signal. Since such a formulation enables imaging with fewer number of measurements that can be provided with $A / D$ converters operating at a fraction of the Nyquist sampling rate or with many less measurements in a stepped frequency systems, it is promising to use compressive sensing especially in high-resolution radar imaging.

Compressive sensing was introduced in the general context of radar imaging in [11], where basic ideas about the possible benefits of CS to radar systems such as lower 
A/D rates, removal of matched filtering in the front end, was outlined. One of the earliest successful examples of using CS in an experimental radar system is demonstrated in [6]. In that work, the subsurface area is modeled to consist of small number of discrete point-like targets. Since the potential targets would cover a small portion of the total subsurface volume to be imaged, the target localization problem was argued to be a sparse recovery problem. Later, a dictionary is generated using a data model for each possible discrete target point, and the sparse subsurface image is generated by solving an optimization problem with CS. Later, these results were expanded for stepped frequency [5] and impulse GPR [7]. In [2], Yoon and Amin. used CS for TWI using wideband beamforming. A technique different than that proposed in [5] was used, where the unmeasured frequency points are reconstructed and conventional wideband beamforming is applied using the reconstructed measurements. In [4], the direct application of what is detailed in [5] is shown for the TWI application; however, a 2-layer world model as in [5] is used which does not quite reflect TWI application since the interaction between backwarding waves and antenna is different when the latter is located over an interface. In [4] simulated images for different measurement matrices are generated, and experimental data results are discussed. In [8] sparse signal reconstruction ideas are applied to GPR rebar detection.

In all previous CS-TWI or CS-GPR literature, the imaging theory depends on several assumptions. First, the targets are point-like reflectors located only at discrete grid positions. Second, the wave velocities or medium parameters, such as permittivity or wall thickness, are assumed to be perfectly known. These assumptions were used to build a forward model between the target space and measurements, but actual targets might not fall on grid points and wave velocities or wall thickness might only be known approximately. Hence the created forward model will definitely include modeling errors. It is very important to understand the effect of such parameters on the robustness of CS-based TWI. Additionally, most CS-TWI papers show simulation results for imaging of randomly placed several point targets with using less space/frequency measurements as compared to observing all space/frequency domain. However, no specific result for subsurface or TWI has been shown detailing the required number of measurements, a function of the sparsity level of the imaged region. The general result of CS, which briefly says $M=O(K \log N)$ is not quite an answer or problem since it only states the order of the measurement number and it is developed for an orthogonal basis case where the dictionary developed for TWI is definitely not orthogonal.

Another important question is what should be the random sampling strategy for CS. This question turns out to be a design of random measurement matrix, which controls how the space and frequency domain is sampled. In [5], randomly selected rows of an identity matrix are used at each antenna position which amounts to measuring random frequency steps. In $[4,7]$ in addition to measuring random frequency steps, a random subsampling in space decreasing the spatial measurement locations was successfully tested.
Also results in $[4,6]$ show that observing less number of uniformly distributed frequencies and spatial measurements generates similar images. However, the tradeoff between decreasing measurements in frequency or space and a good strategy of doing that with how many measurements on each axis needs further investigation. It is also important to know the performance limits of CS-TWI imaging on varying levels of noise.

Range resolution performance of CS-TWI is another important issue. CS has shown to resolve targets spaced closer than the Rayleigh range resolution limit in radar applications [12]. The range resolution performance of CS imaging for TWI application compared to the standard backprojection imaging algorithm is another important point. In this paper a preliminary analysis and results on using CS in TWI for the above-defined challenges are given through numerical simulations. These initial results outline a basis for a more deeper research for given challenges in CS imaging.

The organization of the paper is as follows. Section 2 details the CS-based TWI algorithm, as an extended version of [5] for through-the-wall geometry. Section 3 gives detailed simulation results and analysis about the parameters of the CS-based GPR systems. Conclusions and possible future work are discussed in Section 4.

\section{CS-Based Through-the-Wall Imaging}

Through-the-wall imaging (TWI) algorithms like delay and sum beamforming generate images by mainly applying a coherent matched filter of the measured data with the impulse response of the data acquisition process [13, 14]. Different from the conventional TWI methods, CS-based imaging first discretizes the target space and tries to generate a linear relation between the measured data and the target space to be imaged; hence a data dictionary should be generated. In this dictionary generation each column of the dictionary corresponds to the modeled measurement for a single target at the corresponding grid point in the target space. By this way the measured data can be represented as a linear combination of the dictionary columns. Hence any possible prior information like sparsity could be used.

In this development a stepped frequency (SF) radar system is considered. A detailed explanation on CS-based imaging theory for subsurface imaging for a 2-layer geometry is given in [5] about general stepped frequency systems. Here this development is briefly extended to the TWI application where a 3-layer modeling is considered mostly leaving the details to the original paper.

Our goal is to linearly relate the sensor measurements to the target space. To do so, a target model for which the expected target return can be calculated should be used. Under the Born approximation, neglecting the mutual interactions between targets, the scattering field for a continuous target space $\pi$ can be written as [15]

$$
E_{S}\left(x_{o}, \omega\right)=\iint E_{\mathrm{inc}}\left(x_{0}, w, x, z\right) G\left(x_{0}, w, x, z\right) \pi(x, z) d x d z,
$$


where $E_{S}\left(x_{o}, \omega\right)$ is the scattered field at observation point $x_{o}$, $E_{\text {inc }}$ is the incident field, and $G(\cdot)$ is the relevant Green's function. For this development a discritized version of (2) is basically used. In this development a simple point target modelis selected since the response from a point target can easily be modeled. Other types of target models can also be used if the reflections from it can be modeled. The total target space in this case is assumed to be combination of small number of point targets making the sparsity assumption feasible. This can be viewed as a realistic assumption in many GPR applications like mine detection or TWI where the targets actually cover a small part of the whole target space to be imaged. Assume an SF-GPR acquiring measurements over $P$ targets. The received frequency measurements at the $i$ th scan point can be written as

$$
d_{i}=\sum_{k=1}^{P} r_{k} e^{-j \omega\left(t-\tau_{i}\left(p_{k}\right)\right)},
$$

where $\tau_{i}\left(p_{k}\right)$ is the time delay for the target at the position $p_{k}$ and when the antenna is at the $i$ th scan or observation position. Note that for correct calculation of the time delay from each GPR position to each target position that requires the knowledge of the wave propagation velocity in the medium and sensing geometry. Target reflectivity or other effects are combined in the weights $r_{k}$. The target space $\pi_{T}$ is actually continuous, but to generate a data dictionary it is discretized into a finite set of grid points $T_{S}=\pi_{1}, \pi_{2}, \ldots, \pi_{N}$ where $N$ determines the total number of possible discrete target space points and each $\pi_{j}$ is a $3 \mathrm{D}$ vector $\left[x_{j}, y_{j}, z_{j}\right]$ representing one possible target space point. A data dictionary can be generated by synthesizing the time/frequency data for each possible target space point $\pi_{j}$. Note that actual targets might not be at one of these grid points $\pi_{j}$, but this discretization guides us to build a data dictionary which approximates a linear relation between the target space and measurements. The effect of off-the-grid targets on the imaging performance is analyzed in Section 3.

Finally when the radar is at the $i$ th scan point, the $j$ th column of the dictionary $\Psi_{i}$, corresponding to a target at $\pi_{j}$, can be written as

$$
\left[\Psi_{i}\right]_{j}=\exp \left[-j \omega\left(t-\tau_{i}\left(\pi_{j}\right)\right)\right] .
$$

Repeating (4) for each discrete possible target position creates the dictionary $\Psi_{i}$. This is the dictionary for only the $i$ th scan position. Note that the dimension of $\Psi_{i}$ will be $L \times N$ if $L$ frequency steps are used. Depending on the discritization level, $N$, the possible target points $\pi_{j}$ will be close to the actual target points $p_{k}$. Hence the measured data $d_{i}$ can be represented as a linear combination of the dictionary columns $\left[\Psi_{i}\right]_{j}$ as

$$
d_{i}=\Psi_{i} b+e
$$

where $b$ is a weighted indicator vector defining the target space and $e$ representing any unmodelled factor and noise. From the linear relation defined in (5), the goal is to find $b$ which is actually an image of the medium.
In generating the data dictionary, the crucial point is to calculate the time delay value $\tau_{i}\left(\pi_{j}\right)$ of the propagated wave through the wall and reflected from the point target at position $\pi_{j}$ and received at the receiver when the antennas are at the $i$ th scan point. Exact calculation of this time delay requires the knowledge of wall thickness and wave velocities in all propagated mediums and solution of the diffraction points from the Snell law. Here it is important to note that the wall is assumed to be homogeneous in generating the data dictionary. In general exact knowledge of these parameters is also not available, and effect of the error on these parameters is analyzed on the imaging performance in Section 3. For building the forward model when wave velocities and wall thickness are given, the diffraction points can be solved exactly by the Snell law or linear approximations like [16] or from ray tracing.

Standard stepped frequency systems measure a regularly spaced set of $L$ frequencies in the frequency band they are using. What CS offers is that if the reconstructed signal is sparse then it can be reconstructed with less number of measurements compared to the standard case, thus instead of measuring $L$ frequencies, only a small subset, $M$, of them is measured at each scan point. Here $M<L$. The observed measurements will be $\beta_{i}=\Phi_{i} d_{i}$ where $\Phi_{i}$ is designed to be an $M \times L$ measurement matrix constructed by randomly selecting $M$ rows of an $L \times L$ identity matrix which actually defines which $M$ frequencies out of $L$ are acquired. This effectively reduces the data acquisition time of the $S F$ radar by $L / M$ if all scan points are used. Using measurements $\beta_{i}$ from $S$ different scan positions, the target space $b$ is constructed $[17,18]$ by solving

$$
\hat{b}=\arg \min \|b\|_{1} \quad \text { s.t. }\left\|A^{T}(\beta-A b)\right\|_{\infty}<\epsilon,
$$

where $\beta=\left[\beta_{1}^{T}, \ldots, \beta_{S}^{T}\right]^{T}, \Psi=\left[\Psi_{1}^{T}, \ldots, \Psi_{S}^{T}\right]^{T}, \Phi=$ $\operatorname{diag}\left\{\Phi_{1}, \ldots, \Phi_{S}\right\}$, and $A=\Phi \Psi$. Here the selected $S$ scan positions can also be a random subset of the scan positions within the whole aperture. In (6) selection of the regularization parameter $\epsilon$ is important since it determines the tradeoff between sparsity of the solution and the level of data fit. Selection of the proper relaxation parameter $\varepsilon$ can be done either by estimating the noise variance or using crossvalidation $[19,20]$ techniques which require to separate measurements into two sets and recurrent solutions of the optimization problem. In the presented numerical results, an empirical selection of this parameter as $\epsilon=0.2 * A^{T} \beta$ is done. An optimization package CVX [21] is used for solution of (6) for the numerical simulation results presented in the next section.

\section{Numerical Simulations and Results}

In this section the effects of the parameters in CS imaging system are tested through numerical simulations. A preliminary understanding on the required number of measurements for correct reconstruction of the target space at varying sparsity levels, the effect of acquiring these measurements from frequency or space, the level of noise where CS imaging still works, the effect of system bandwidth 
and resolution limits, the effect of discritization of target space and off-the-grid targets, and the estimation errors in parameters like wave velocity or wall thickness in TWI are aimed in the simulations presented in this section. For these tests a TWI geometry with a monostatic antenna with a $20 \mathrm{~cm}$ offset from a $30 \mathrm{~cm}$ thick wall of dielectric constant 4 is considered. The SF system collects frequency domain measurements from $500 \mathrm{MHz}$ to $5.5 \mathrm{GHz}$ with $40 \mathrm{MHz}$ frequency steps. Thus, at each scan position, GPR acquires 126 frequency measurements if all frequency steps are measured. A target space of size $60 \mathrm{~cm}$ by $60 \mathrm{~cm}$ is considered with a $3 \mathrm{~cm}$ discretization on both axes generating an $N=400$ total grid point. Unless stated targets are assumed to be point like and exactly positioned on grid locations.

3.1. Effect of Measurement Number. In CS-based subsurface imaging or TWI literature, it is shown that the total number of measurements acquired can be decreased if the target space is sparse, but it is important to know the relation between the required number of measurements for correct imaging and varying levels of sparsity specifically for GPR applications. To understand this relation, target spaces with sparsity levels changing from 2 to 12 are tested. For each case the target space is imaged with 10 to 500 compressed measurements using proposed CS technique. This procedure is repeated 50 times for each sparsity level and measurement number. For each case a random target space and random measurement selections are done and correct reconstructions are counted. The correct reconstruction ratio is obtained as the ratio of total number of correct reconstructed cases to the total number of tests which is 50 in this case. Two measurement strategies as random and uniform are compared. In the random measurement strategy acquiring $M$ measurement simply means $M$ of the total $L \times S$ space-frequency measurement are randomly selected. The uniform measurement strategy takes this $M$ measurements as uniformly spaced out of $L S \times 1$ measurement vector. Note that standard back-projection techniques mainly use all $L \times S$ space-frequency measurements.

Figures 1(a) and 1(b) show the correct reconstruction ratio at the acquired measurement numbers for varying levels of sparsity for random and uniform measurement strategies, respectively. It can be observed that both strategies perform similarly. This result also confirms the observation done in $[4,6]$. The results in Figures $1(\mathrm{a})$ and $1(\mathrm{~b})$ also show that for correct reconstruction required number of measurements should increase as the sparsity level increases. Figure 1(c) shows the required number of measurements to achieve a 95\% correct reconstruction ratio for each tested sparsity level for this numerical simulation. It is observed that the the required number of measurements linearly increases with the sparsity level of the solution which is in accordance with the fundamental result of CS which states that the required number of measurements should be $M=$ $O(K \log N)$ which states a relation as $M=c K \log N$ with an unknown constant $c$. A first-order fit applied to the result in Figure $1(\mathrm{c})$ gives a relation as $M \approx 16 K$, and when the dimension of the target space $N=400$ is considered, the required number of measurements for this case can be written as $M \approx 2.7 \mathrm{~K} \log N$ where the unknown constant $c$ is found to be 2.7 for the tested CS-TWI scenario. This relation states the minimum number of compressive measurements to correctly reconstruct a $K$ sparse scene using our model, and note that it is developed for a specific case of $N=400$. We have tested this relation with a similar scenario, but using a discritization of $N=800$ for various sparsity levels and in the average a correct reconstruction ratio of 0.96 is obtained. It is important to note that this result is dependent on the dictionary developed in Section 2. We do not conclude that this is a general result for any CS-TWI case specifying the minimum required number of measurements, but we believe this relation would at least give an idea on that.

\subsection{Effect of Acquiring Measurements from Frequency or} Space. Another important point in a measurement strategy is to decide on a good division in observing measurement from frequency or space. It is important to understand the tradeoff between subsampling from space or frequency. As an example, if a total of 50 measurement would be taken from the whole space-frequency domain how would you distribute the measurements to space and frequency; that is, would you take random 10 scan points and measure 5 frequencies at each scan point or measure 5 scan points and take 10 frequency measurements at each point. Which one would be more effective in reconstructing the target space image? For this test the target sparsity level is fixed to 5 targets, and varying numbers of randomly selected frequency measurements from 5 to 125 at varying spatial scan points from 2 to 20 are observed. At each case the independent target space generations and random measurement selections are repeated 50 times and the number of correct reconstructions are counted. Figure 2 shows the correct reconstruction ratio by the measurement number at each scan point. Note that the $x$-axis in Figure 2 indicates the measurement number per each scan point out of a maximum of 126 frequency measurements with the simulation parameters used.

It can be initially observed that imaging performance is very low if only one scan point is used regardless of the measurement number, but even increasing it to two scan points increases the performance. For the same total spacefrequency measurement numbers, distributing measurements across scan points increases correct reconstruction performance. For example, observing 90 measurements at a single scan point generates a 0.1 reconstruction ratio where observing 40 measurements at two scan points or 30 measurements at 3 scan points increases reconstruction performance dramatically. It can also be observed that the total number of measurements becomes the important factor if at least 4 out of 20 scan points are used. As a conclusion, random subsampling in both frequency and space can be done but for successful reconstructions spatial scan points should not be too few. Further and more general tests are needed to be done to obtain more general results including whether this tradeoff between space and frequency division is effected by the sparsity level of the scene. 


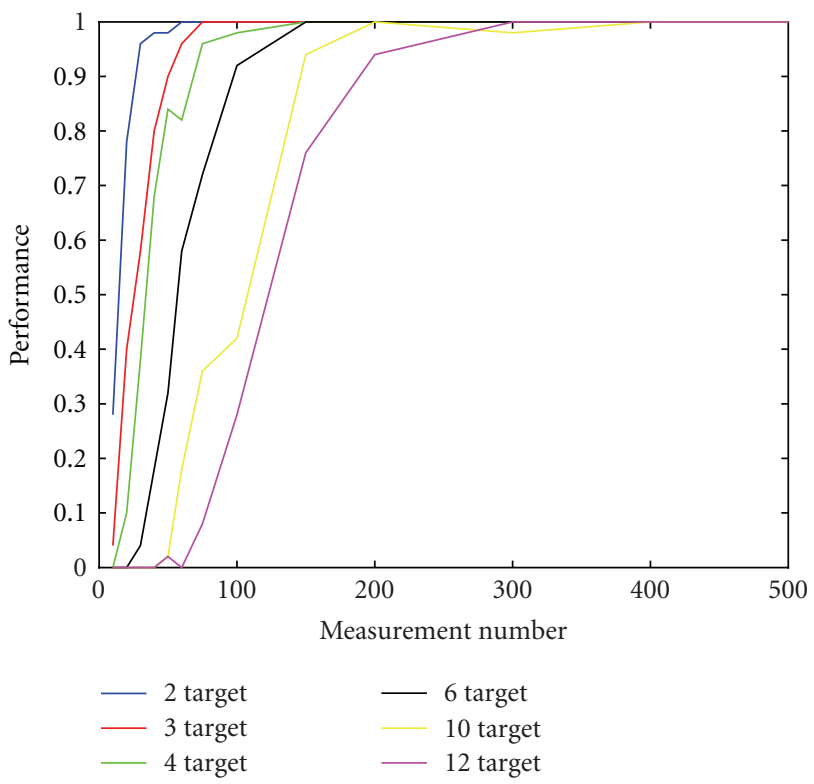

(a)

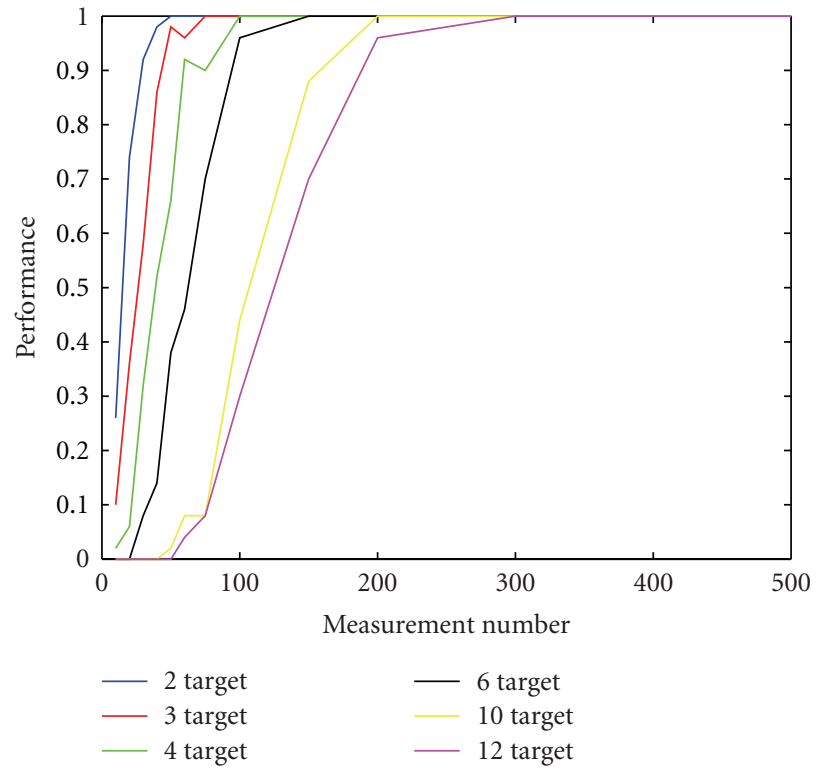

(b)

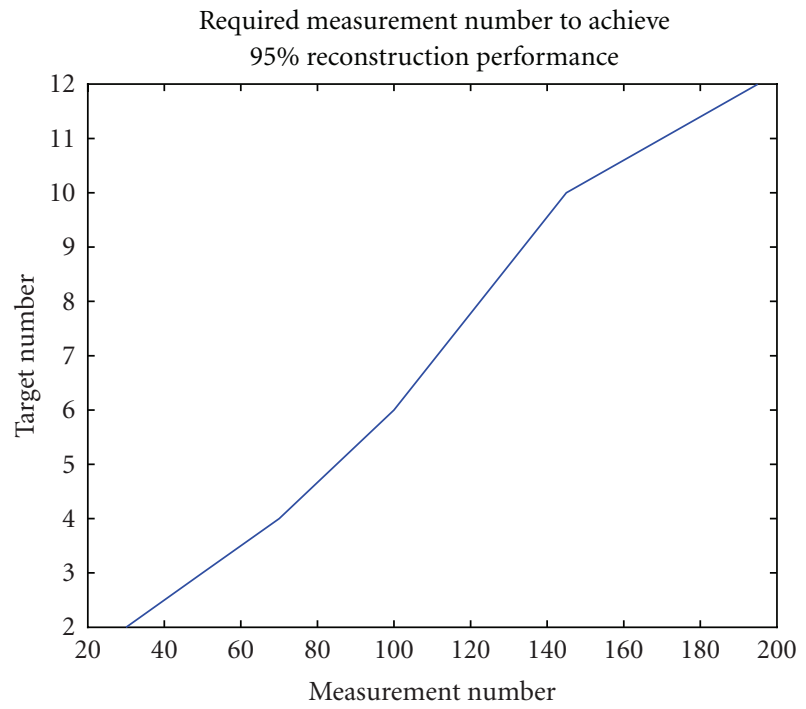

(c)

FIGURE 1: Correct reconstruction ratio versus measurement number for varying levels of target sparsity for (a) random measurement strategy, (b) uniform measurement strategy. The required number of measurements to achieve a 95\% correct reconstruction for random measurement strategy.

3.3. Effect of Noise Level. To analyze the impact of noise level on the imaging performance, a simulation is performed. First, with frequency domain data for a single point target SNRs from -5 to $25 \mathrm{~dB}$ are tested with 50 different trials using additive complex white gaussian noise (CWGN) at each SNR level using varying number of total of spacefrequency measurements from 50 to 300 observed randomly at 20 scan points and the target space is reconstructed with (6). Figure 3 shows the correct reconstruction ratio versus SNR for the tested measurement numbers. The regularization parameter is empirically used as $\epsilon=0.2 * A^{T} \beta$ for all cases.

It can be seen in simulation result that with increasing measurement numbers, the same reconstruction performance could be obtained at lower SNR values. At lower SNR values than $0 \mathrm{~dB}$, correct reconstructions were not obtained for the tested measurement numbers. Above $15 \mathrm{~dB}$ SNR, even very low measurement numbers generate highly correct reconstruction ratios.

3.4. Effect of Bandwidth and Enhanced Range Resolution on CS. One of the important properties of the CS method is its ability to resolve targets spaced closer than the conventional range resolution of an SFCW GPR [5, 22], defined as $\Delta R=$ $c /(2 L \Delta f)$, where $\Delta f$ is the frequency step, $L$ is the number of frequency steps, and $c$ is the speed of the wave in the medium. To study this increased resolution property for GPR applications, a simulation is done with two point targets. In the simulation a bandwidth of $2.5 \mathrm{GHz}$ is used with starting 


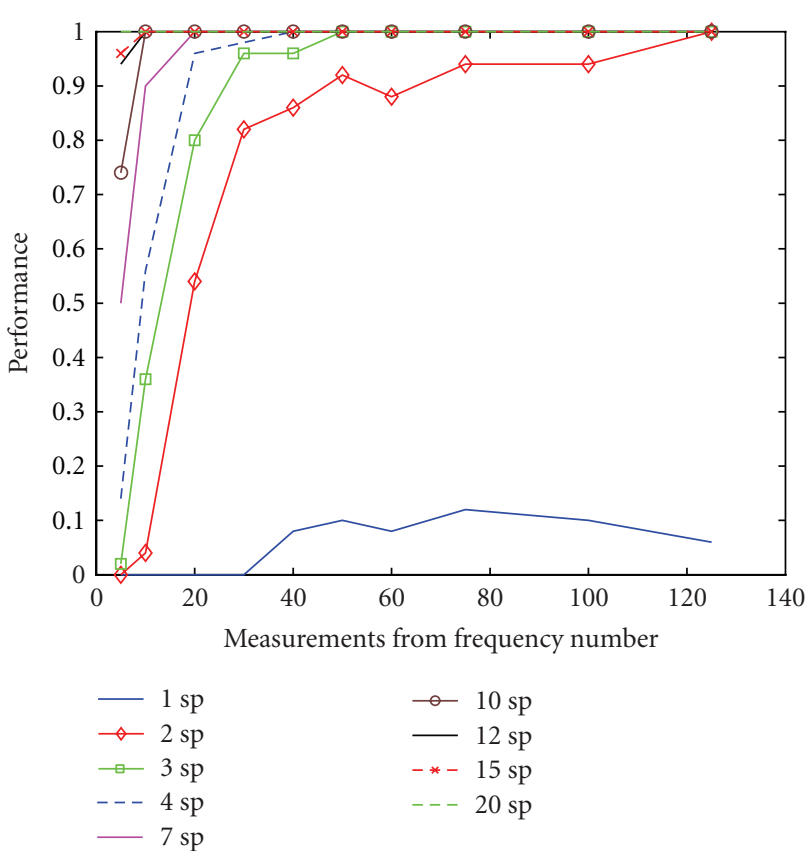

FIGURE 2: Correct reconstruction ratio versus the random frequency measurements per each scan point. The term "sp" in legend represents "scan point."

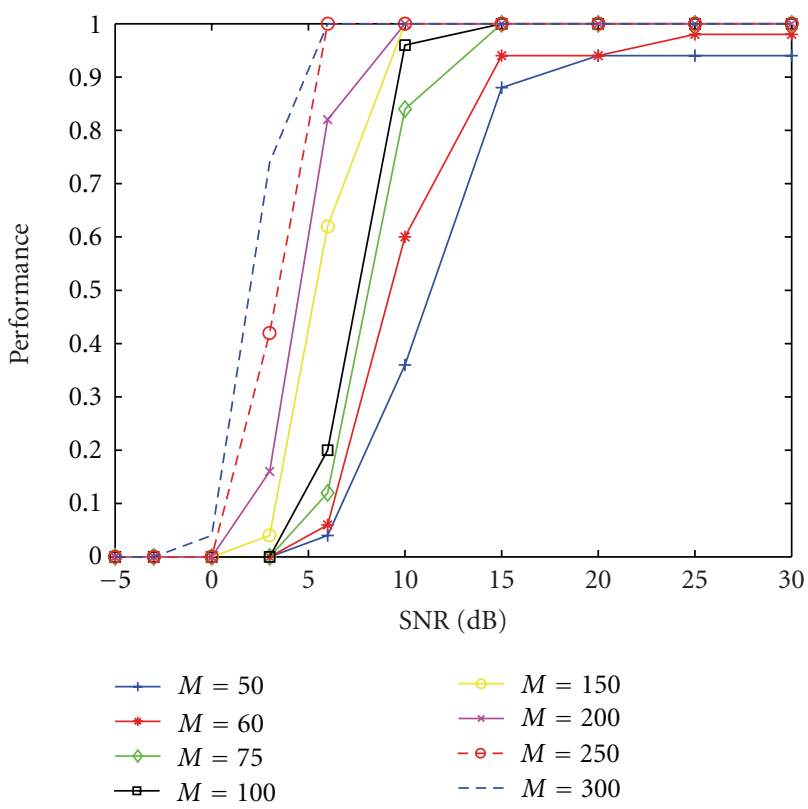

FIGURE 3: Correct reconstruction ratio versus SNR for different total measurement number $M$.

and ending frequencies of $0.5 \mathrm{GHz}$ and $3 \mathrm{GHz}$, respectively. Hence, in theory, range resolution is going to be $6 \mathrm{~cm}$. The target space is discritized to $1 \mathrm{~cm}$ for this simulation. Figures $4(\mathrm{a})$ and 4(b) show the delay and sum beamforming results when the separation between two targets is $10 \mathrm{~cm}$ and $6 \mathrm{~cm}$, respectively. As expected the two targets could be located correctly when their separation is more than the resolution limit. While delay and sum beamforming cannot resolve the

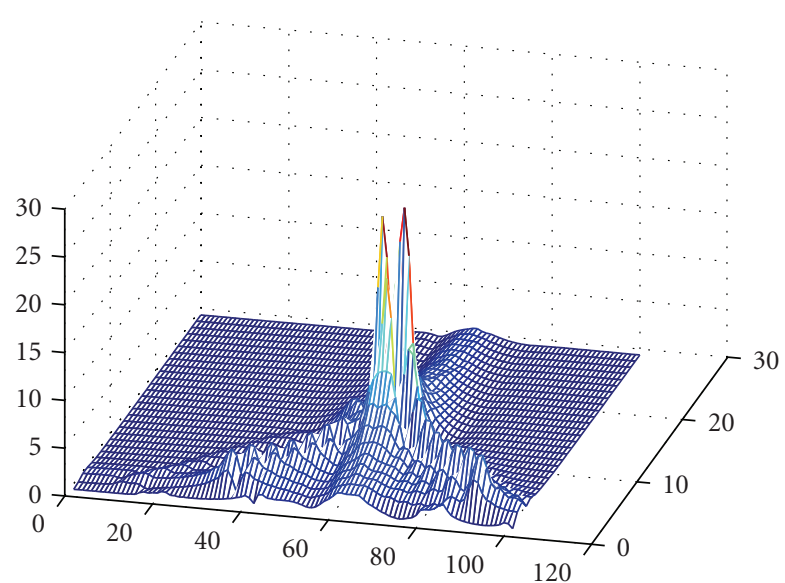

(a)

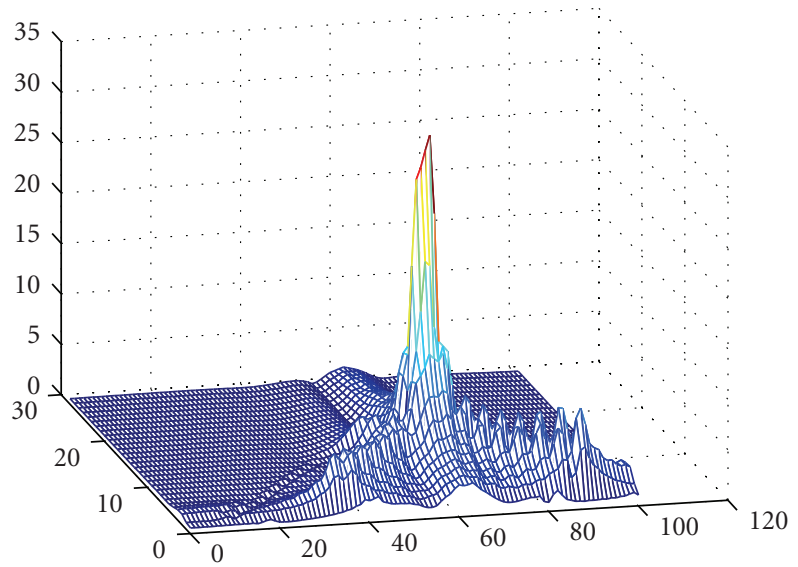

(b)

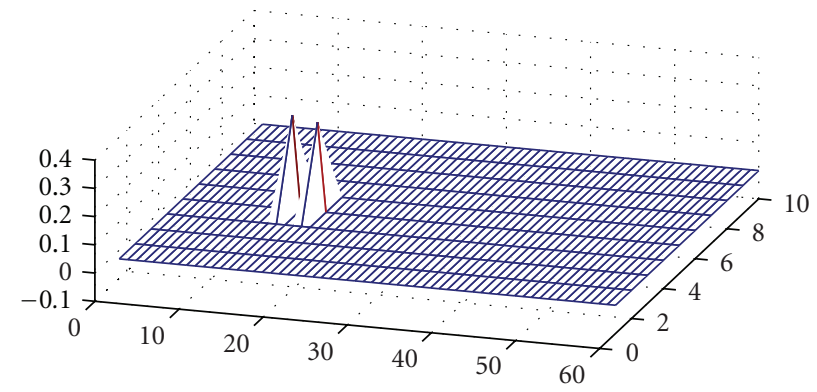

(c)

FIGURE 4: Imaging performance of backprojection when the range separation between two targets is (a) $10 \mathrm{~cm}$, (b) $6 \mathrm{~cm}$, and (c) performance of CS-based imaging when two targets are separated by $3 \mathrm{~cm}$.

two targets because they merge into a single peak for closer targets, the CS method resolves both targets at their correct positions even if the separation between them is $3 \mathrm{~cm}$ in range which is smaller than the range resolution limit. This is because the CS method does not perform a matched filtering operation, which would impose the range resolution limit. Instead CS tries to explain the measurements using the least number of columns from its dictionary of measurements. The resolution limit in CS is mainly about the mutual coherence of the dictionary columns and the regularization 
parameter used. The increased resolution property of CS has also been reported in other sparse signal reconstruction applications $[23,24]$.

3.5. Effect of Off-the-Grid Target. In the GPR imaging applications, the assumed signal sparsity is in the continuous target space and the sparsity basis $\Psi$ is constructed through discritization or gridding of this target space. In a general setting, the observed signal will not be sparse in such a dictionary created through discritization since a target might not be exactly on any of the grid locations. The reconstruction performance with CS will decrease due to this basis mismatch. This is not a specific problem for GPR, but it is general for any reconstruction problem where a parametric continuous space is sensed and similar basis mismatch or off-grid problems are heavily pronounced in radar imaging [25] for delay-Doppler space, target localization $[5,26]$ for continuous target space, beamforming [27, 28] for angle space, or shape detection [29] for shape parameter space. Such examples emphasize the importance of off-the-grid problem in CS.

In the literature the simplistic and the general approach to the problem is to increase the grid size and use a reconstruction algorithm that can handle additive noise in measurements. Although this could provide close to satisfactory results for some applications, it is not the solution to the actual problem. No matter how fine the discritization is, the actual signal parameters might not be on the grid. In addition increasing grid size increases the coherence between dictionary columns making restricted isometry property (RIP) [30] invalid. Grid size increase also increases the computational complexity of reconstruction. It is also important to note that the modeling error due to basis mismatch is also not an additive noise; it is rather multiplicative [31].

In this part, off-the-grid targets are simulated, and effect of the discritization or grid size on the TWI performance is observed. To observe only the effect of grid size, parameters like wall thickness or wave velocity in all mediums are assumed to be known perfectly. A target space of size $60 \times$ $60 \mathrm{~cm}^{2}$ area is simulated. Two point reflectors at off-the-grid positions as $(-0.192,0,-0.143)$ and $(0.218,0,-0.382)$ are placed, and the target space are discritized with $1 \mathrm{~cm}, 2 \mathrm{~cm}$, and $5 \mathrm{~cm}$ grid sizes. An SNR of $10 \mathrm{~dB}$ is used. The obtained compressive sensing and back-projection reconstructions using only the $20 \%$ of the total frequency-space data randomly are shown in Figure 5 for each discritization level.

It can be observed from Figure 5 that CS-based imaging method is effected from off-the-grid targets and the imaging performance gets worse if the grid sizes get high. For grid size of $1 \mathrm{~cm}$, both targets are observed with some additional targets, but, for higher grid sizes of 2 and $5 \mathrm{~cm}$ the created images are not close to correct target space image. Back-projection images in general can be argued to be more robust to discritization of target space compared to compressive-sensing-based imaging technique. But performance of backprojection also gets worse as the grid sizes increase. These results show that off-the-grid targets are really an important problem for CS-based techniques. Although the simulation indicates that off-grid-targets create challenges for CS; in real-world scenarios possible extended targets would cover several grid locations and the observed data could be represented sparsely with those grid locations, which is mainly observed with the experimental CS based imaging results in the literature. Nevertheless depending on the grid size, off-the grid targets creates challenges. This discussion brings a new data model. The signal is actually sparse in an unknown basis $\widehat{\Phi}$ where there is mismatch between the actual sparsity basis $\widehat{\Phi}$ and the modeled one $\Phi$. In the literature there are several works $[31,32]$ analyzing the effects of stated basis mismatch problem. However, these works only study the sensitivity of CS to the mismatch between the assumed and actual sparsity bases and calculate resultant performance degradation. Analytical $\ell_{2}$ norm error bounds due to the basis mismatch are also obtained in $[31,32]$. They do not offer any systematic solution to the problem.

To solve the off-grid target problem, we are working on new techniques that utilize gradients with respect to the parameters at each grid location and apply greedy or joint perturbations to basis columns. This solution for the offgrid problem is combined with the orthogonal matching pursuit (OMP) algorithm for a new iterative off-the-grid OMP method. Our initial results are given in [33] but extended techniques will be applied to the GPR applications and its performance will also be analyzed in the future.

3.6. Effect of Unknown Parameters. Another fundamental problem in CS-based TWI is that the parameters like wall thickness and the velocity of the wave in the wall that are used in creating the data dictionary might be different than the actual parameters. These problems are also studied in classical TWI in $[34,35]$. Although it is possible to estimate these parameters, still it is very hard to exactly find them. Here the effect of the errors in these estimated parameters on the CS imaging is shown. For the presented results the targets are placed at exactly on-the-grid points, and only effects of the errors in wall thickness and wave velocity are analyzed. The data dictionary is generated using a wall of thickness of $30 \mathrm{~cm}$ and permittivity 4 . Figures 6(a) and 6(b) show the images created when the actual wall permittivity is taken as 4.1 and 4.5, respectively. When the error is small, two targets are imaged at their correct positions, but when the error gets large the created image deviates highly from the correct image. When the measurements are simulated with a wall thickness of $30.2 \mathrm{~cm}$, even this small error in wall thickness creates an important degradation in the CS image. When the wall thickness is taken as $31 \mathrm{~cm}$, the created image is not related with the correct target space. Hence CS-based imaging seems to be highly dependent on close estimation of the unknown parameters. On the other hand, similar tests are done on backprojection, and it is observed that with even higher errors in parameters, backprojected images are not effected as much as CS-based images. It can be stated that backprojection is also more robust to errors in modeling parameters. This could be because backprojection 


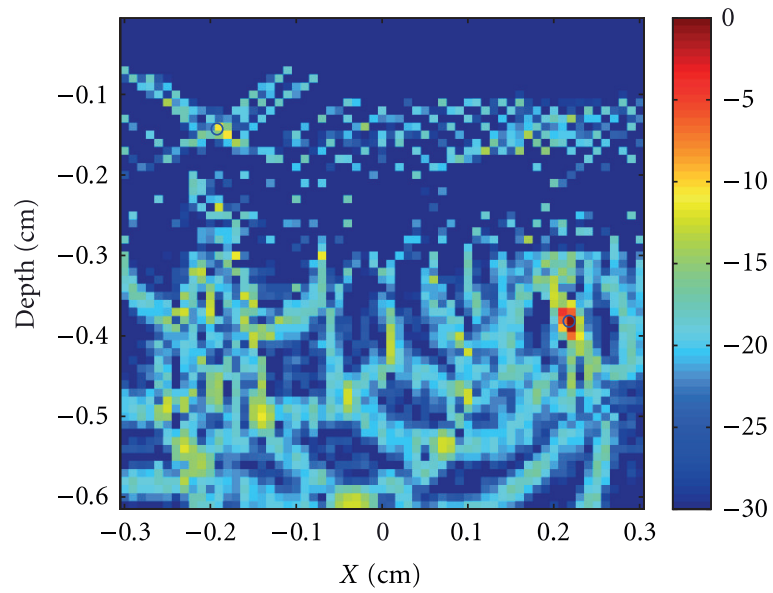

(a)

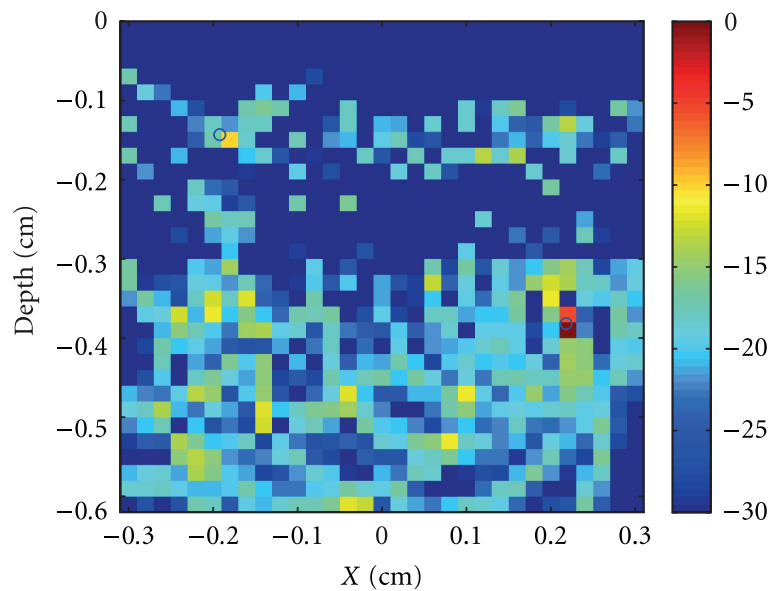

(c)

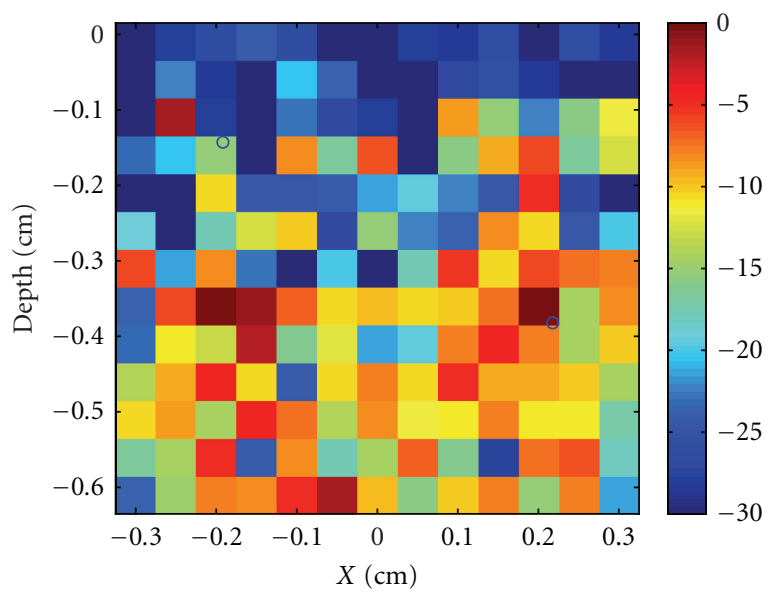

(e)

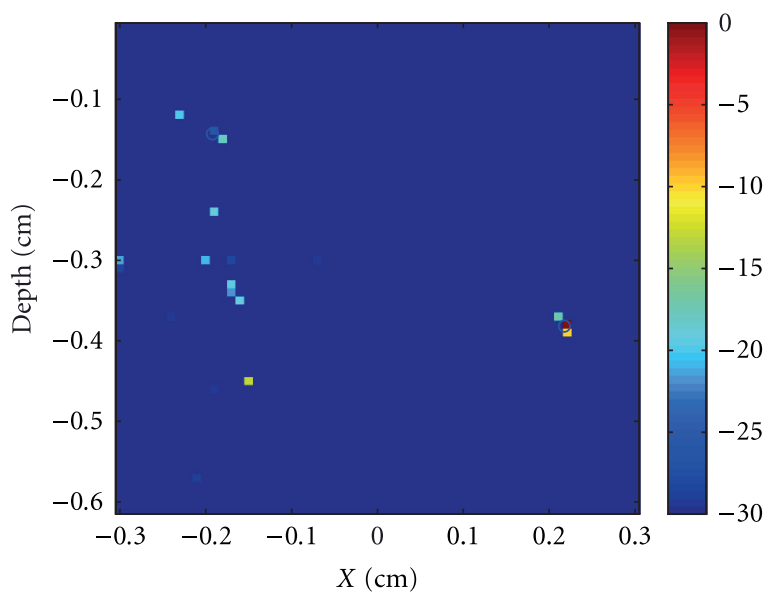

(b)

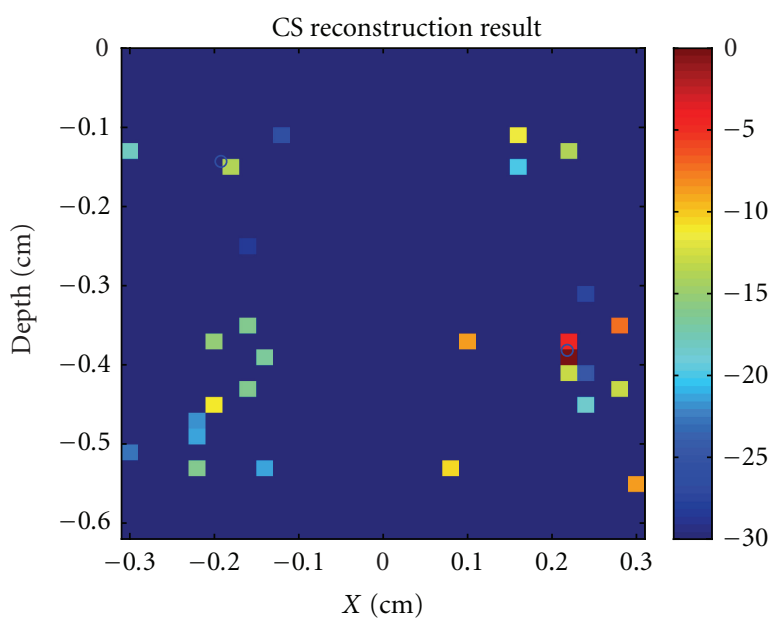

(d)

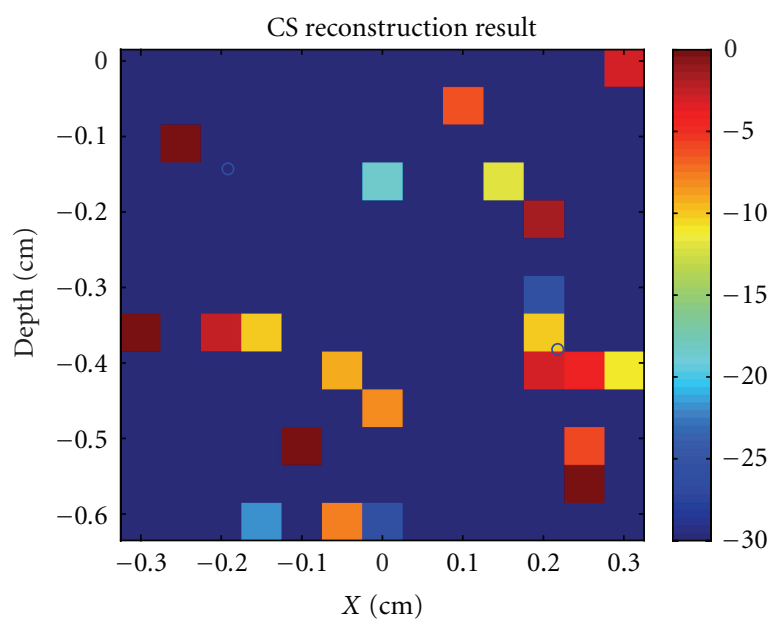

(f)

FIGURE 5: Effects of off-the-grid targets to the CS-based TWI: (a) backprojection, (b) CS image for discritization of $1 \mathrm{~cm}$, (c) backprojection, (d) CS image for discritization of $2 \mathrm{~cm}$, (e) backprojection, (f) CS image for discritization of $5 \mathrm{~cm}$. 


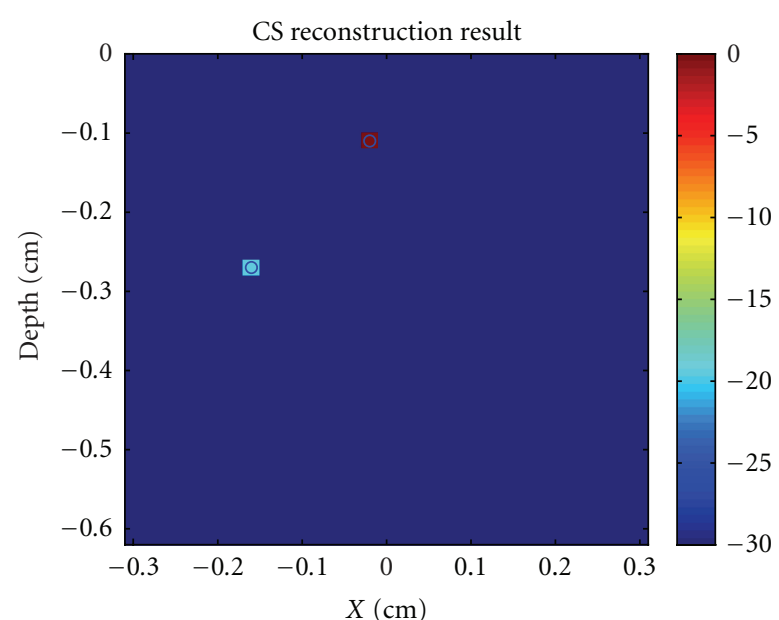

(a)

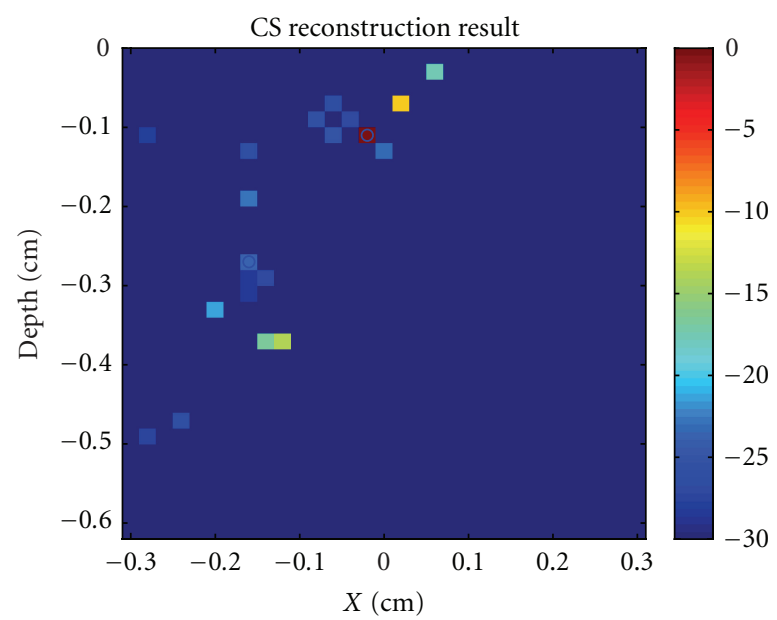

(c)

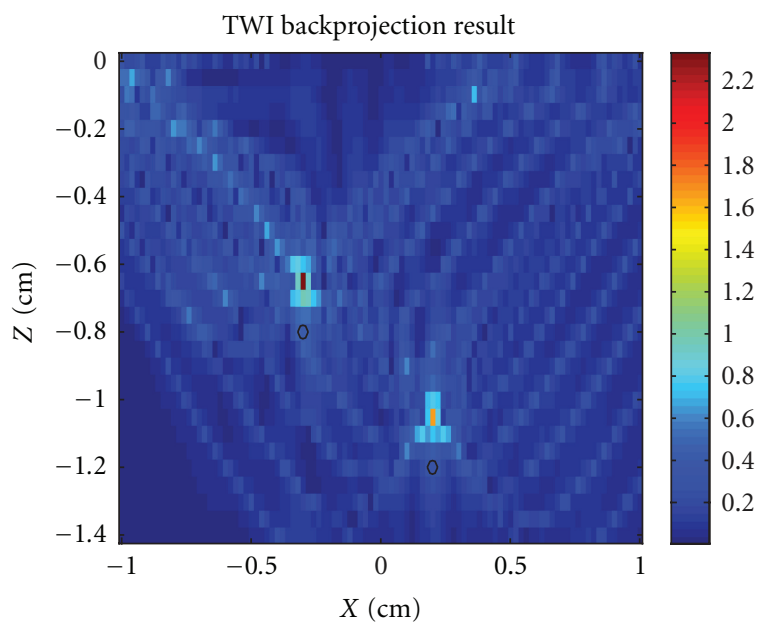

(e)

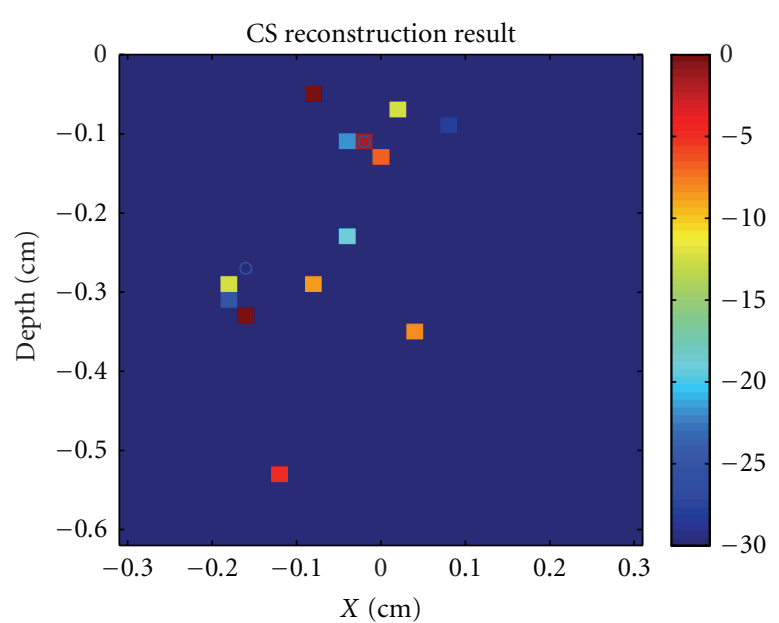

(b)

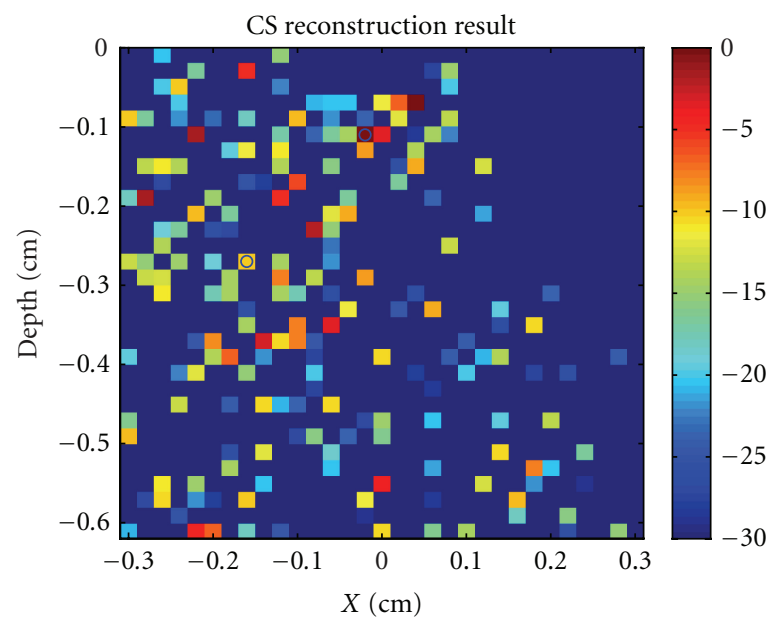

(d)

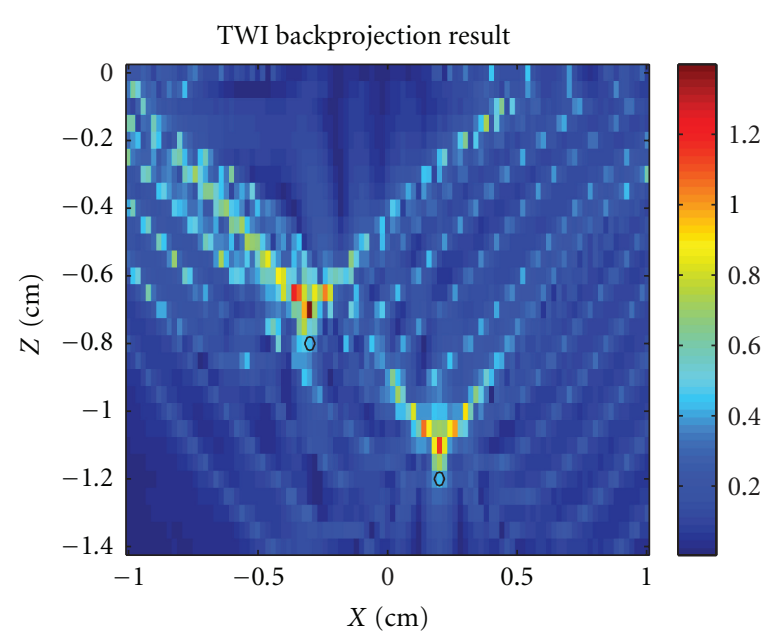

(f)

FIGURE 6: Effects of unknown wall characteristics to CS imaging: wall permittivity is (a) 4.1 and (b) 4.5 and wall thickness (c) $30.2 \mathrm{~cm}$ and (d) $31 \mathrm{~cm}$. Effects of unknown wall characteristics to back-projection imaging: wall permittivity is (e) 4.7 and Wall thickness (f) $33 \mathrm{~cm}$. 
only calculates the correlation of the measurements with the data model for the grid location and focused and highly correlated but not sparse images are obtained. On the other hand, CS tries to match the measurements with linear combinations of the dictionary columns. In the presence of modeling errors and also depending on the regularization parameter, CS brings new data points to satisfy the data constraint. Hence it is very important to generate either successful parameter estimation techniques that use only compressive measurements or more robust sparsityenhanced imaging techniques. Extending data dictionaries using several different parameters [36] might be an initial solution although it increases the imaging complexity or possible gradient-based search parameter search techniques could be used.

\section{Conclusion}

In this paper, a compressive-sensing-based through-thewall imaging algorithm is presented. Initial results through numerical simulations are obtained for questions on the required number of measurements for a sparsity level, measurement strategy to subsample in frequency and space, imaging performance in varying noise levels, and enhanced CS range resolution performance. Simulations with off-thegrid targets and unknown parameters are performed, and it is observed that if the grid size or the error in the unknown parameters is not too big, the imaging performance is not severely affected; however, big grid sizes or errors degrade the reconstructed image. Although obtained results are not too general, we believe that they are preliminary to a much deeper study.

\section{Acknowledgments}

This work was supported by the Scientific and technical Research Council of Turkey (TUBITAK) under Contract Agreement 109E280 and within the Marie Curie IRG Grant with Grant Agreement PIRG04-GA-2008-239506.

\section{References}

[1] M. G. Amin, Through-The-Wall Radar Imaging, CRC Press, Boca Raton, Fla, USA, 2010.

[2] Y. S. Yoon and M. G. Amin, "Imaging of behind the wall targets using wideband beamforming with compressive sensing," in Proceedings of the IEEE/SP 15th Workshop on Statistical Signal Processing (SSP '09), pp. 93-96, September 2009.

[3] Y. S. Yoon and M. G. Amin, "Through-the-wall radar imaging using compressive sensing along temporal frequency domain," in Proceedings of the IEEE International Conference on Acoustics, Speech, and Signal Processing (ICASSP '10), pp. 28062809, March 2010.

[4] Q. Huang, L. Qu, B. Wu, and G. Fang, "UWB through-wall imaging based on compressive sensing," IEEE Transactions on Geoscience and Remote Sensing, vol. 48, no. 3, pp. 1408-1415, 2010.

[5] A. C. Gurbuz, J. H. McClellan, and W. R. Scott Jr., "A compressive sensing data acquisition and imaging method for stepped frequency GPRs," IEEE Transactions on Signal Processing, vol. 57, no. 7, pp. 2640-2650, 2009.

[6] A. C. Gurbuz, J. H. McClellan, and W. R. Scott Jr., "Compressive sensing for GPR imaging," in Proceedings of the 41st Asilomar Conference on Signals, Systems and Computers (ACSSC '07), pp. 2223-2227, November 2007.

[7] A. C. Gurbuz, J. H. McClellan, and W. R. Scott Jr., "Compressive sensing for subsurface imaging using ground penetrating radar," Signal Processing, vol. 89, no. 10, pp. 1959-1972, 2009.

[8] F. Soldovieri, R. Solimene, L. Lo Monte, M. Bavusi, and A. Loperte, "Sparse reconstruction from GPR data with applications to rebar detection," IEEE Transactions on Instrumentation and Measurement, vol. 60, no. 3, pp. 1070-1079, 2011.

[9] D. L. Donoho, "Compressed sensing," IEEE Transactions on Information Theory, vol. 52, no. 4, pp. 1289-1306, 2006.

[10] E. J. Candès, J. Romberg, and T. Tao, "Robust uncertainty principles: exact signal reconstruction from highly incomplete frequency information," IEEE Transactions on Information Theory, vol. 52, no. 2, pp. 489-509, 2006.

[11] R. Baraniuk and P. Steeghs, "Compressive radar imaging," in Proceedings of the IEEE Radar Conference, pp. 128-133, April 2007.

[12] M. A. Herman and T. Strohmer, "High-resolution radar via compressed sensing," IEEE Transactions on Signal Processing, vol. 57, no. 6, pp. 2275-2284, 2009.

[13] E. J. Baranoski, "Through-wall imaging: historical perspective and future directions," Journal of the Franklin Institute, vol. 345, no. 6, pp. 556-569, 2008.

[14] F. Ahmad, Y. Zhang, and M. G. Amin, "Three-dimensional wideband beamforming for imaging through a single wall," IEEE Geoscience and Remote Sensing Letters, vol. 5, no. 2, pp. 176-179, 2008.

[15] F. Soldovieri and R. Solimene, "Through-wall imaging via a linear inverse scattering algorithm," IEEE Geoscience and Remote Sensing Letters, vol. 4, no. 4, pp. 513-517, 2007.

[16] E. M. Johansson and J. E. Mast, "Three-dimensional groundpenetrating radar imaging using synthetic aperture timedomain focusing," in Advanced Microwave and MillimeterWave Detectors Conference, vol. 2275 of Proceedings of SPIE, pp. 205-214, July 1994.

[17] E. J. Candès, J. K. Romberg, and T. Tao, "Stable signal recovery from incomplete and inaccurate measurements," Communications on Pure and Applied Mathematics, vol. 59, no. 8, pp. 1207-1223, 2006.

[18] D. L. Donoho, M. Elad, and V. N. Temlyakov, "Stable recovery of sparse overcomplete representations in the presence of noise," IEEE Transactions on Information Theory, vol. 52, no. 1, pp. 6-18, 2006.

[19] R. Ward, "Cross validation in compressed sensing via the Johnson Lindenstrauss Lemma," 2008.

[20] P. Boufounos, M. F. Duarte, and R. G. Baraniuk, "Sparse signal reconstruction from noisy compressive measurements using cross validation," in Proceedings of the IEEE/SP 14th WorkShoP on Statistical Signal Processing (SSP '07), pp. 299-303, August 2007.

[21] M. Grant and S. Boyd, "Cvx: Matlab softwarefor disciplined convex programming (web page and software)," http://www .cvxr.com/cvx/.

[22] S. O. Piper, "Homodyne FMCW radar range resolution effects with sinusoidal nonlinearities in the frequency sweep," in Proceedings of the IEEE International Radar Conference, pp. 563-567, May 1995. 
[23] D. L. Donoho, "Superresolution via sparsity constraints," SIAM Journal on Mathematical Analysis, vol. 23, no. 5, pp. 1309-1331, 1992.

[24] D. Malioutov, M. Çetin, and A. S. Willsky, "A sparse signal reconstruction perspective for source localization with sensor arrays," IEEE Transactions on Signal Processing, vol. 53, no. 8, pp. 3010-3022, 2005.

[25] J. H. G. Ender, "On compressive sensing applied to radar," Signal Processing, vol. 90, no. 5, pp. 1402-1414, 2010.

[26] V. Cevher, M. Duarte, and R. Baraniuk, "Distributed target localization via spatial sparsity," in Proceedings of the European Signal Processing Conference (EUSIPCO '08), pp. 134-142, August 2008.

[27] A. C. Gurbuz, V. Cevher, and J. H. McClellan, "A compressive beamformer," in Proceedings of the IEEE International Conference on Acoustics, Speech, and Signal Processing (ICASSP '08), Las vegas, Nev, USA, April 2008.

[28] Y. Yu, A. P. Petropulu, and H. V. Poor, "MIMO radar using compressive sampling," IEEE Journal on Selected Topics in Signal Processing, vol. 4, no. 1, pp. 146-163, 2010.

[29] N. Aggarwal and W. C. Karl, "Line detection in images through regularized hough transform," IEEE Transactions on Image Processing, vol. 15, no. 3, pp. 582-591, 2006.

[30] R. Baraniuk, M. Davenport, R. DeVore, and M. Wakin, "A simple proof of the restricted isometry property for random matrices," Constructive Approximation, vol. 28, no. 3, pp. 253263, 2008.

[31] M. A. Herman and T. Strohmer, "General deviants: an analysis of perturbations in compressed sensing," IEEE Journal on Selected Topics in Signal Processing, vol. 4, no. 2, pp. 342-349, 2010.

[32] L. Scharf, Y. Chi, A. Pezeshki, and R. Calderbank, "Sensitivity to basis mismatch in compressed sensing," in Proceedings of the IEEE International Conference on Acoustics, Speech, and Signal Processing (ICASSP '10), pp. 3930-3933, March 2010.

[33] O. Teke, A. C. Gurbuz, and O. Arikan, "A new omp techniques for sparse recovery," in Proceedings of the 20. IEEE Sinyal isleme ve iletisim Uygulamalari (SIU) Kurultayi, Fethiye, Turkey, 2012.

[34] G. Mandapati, F. Ahmad, and M. G. Amin, "Autofocusing of through-the-wall radar imagery under unknown wall characteristics," IEEE Transactions on Image Processing, vol. 16, no. 7, pp. 1785-1795, 2007.

[35] Y. Zhang, G. Wang, and M. G. Amin, "New approach for target locations in the presence of wall ambiguities," IEEE Transactions on Aerospace and Electronic Systems, vol. 42, no. 1, pp. 301-315, 2006.

[36] M. A. C. Tuncer and A. C. Gurbuz, "Analysis of unknown velocity and target off the grid problems in compressive sensing based subsurface imaging," in Proceedings of the IEEE International Conference on Acoustics, Speech, and Signal Processing (ICASSP '11), pp. 2880-2883, Prague, Czech Republic, 2011. 

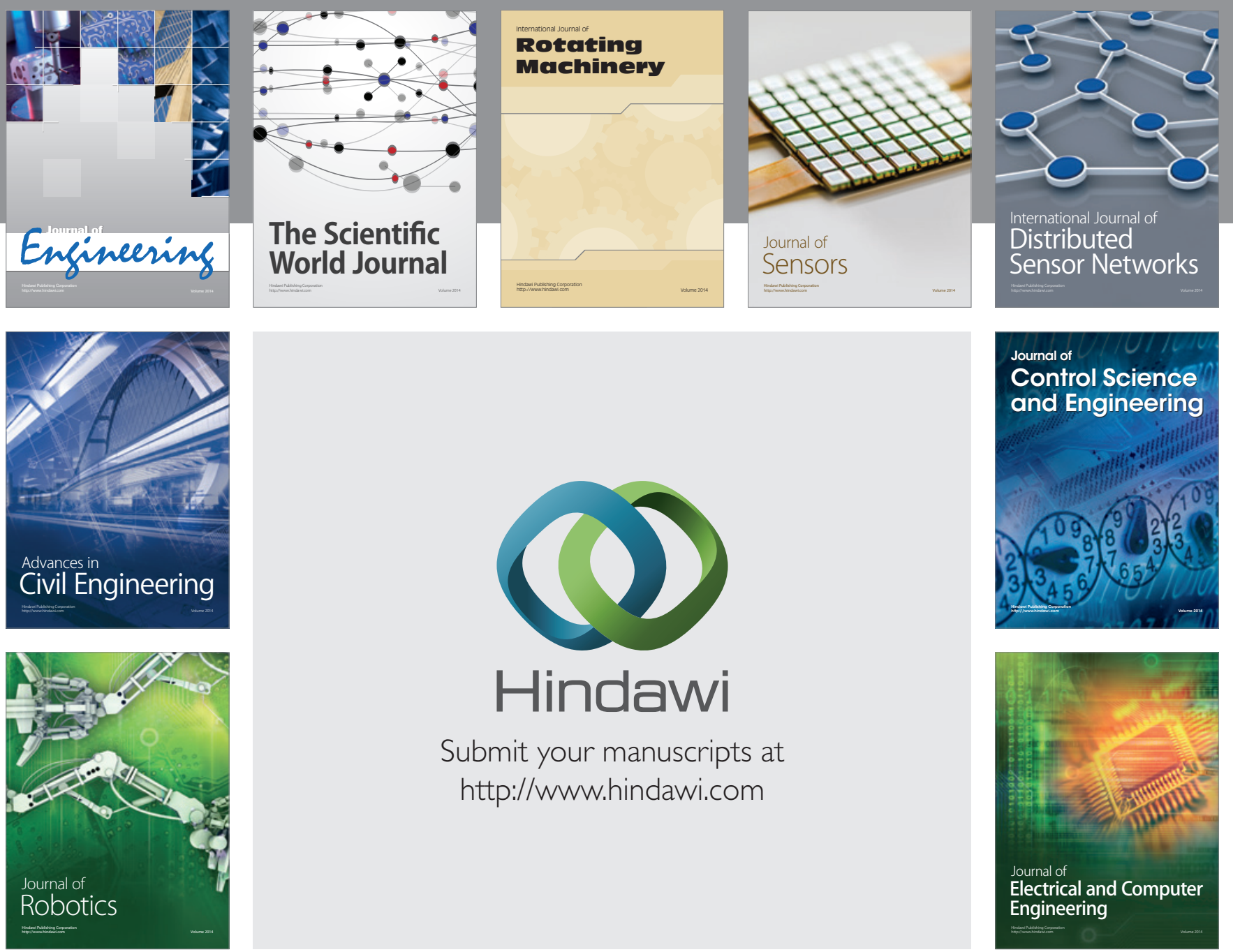

Submit your manuscripts at

http://www.hindawi.com
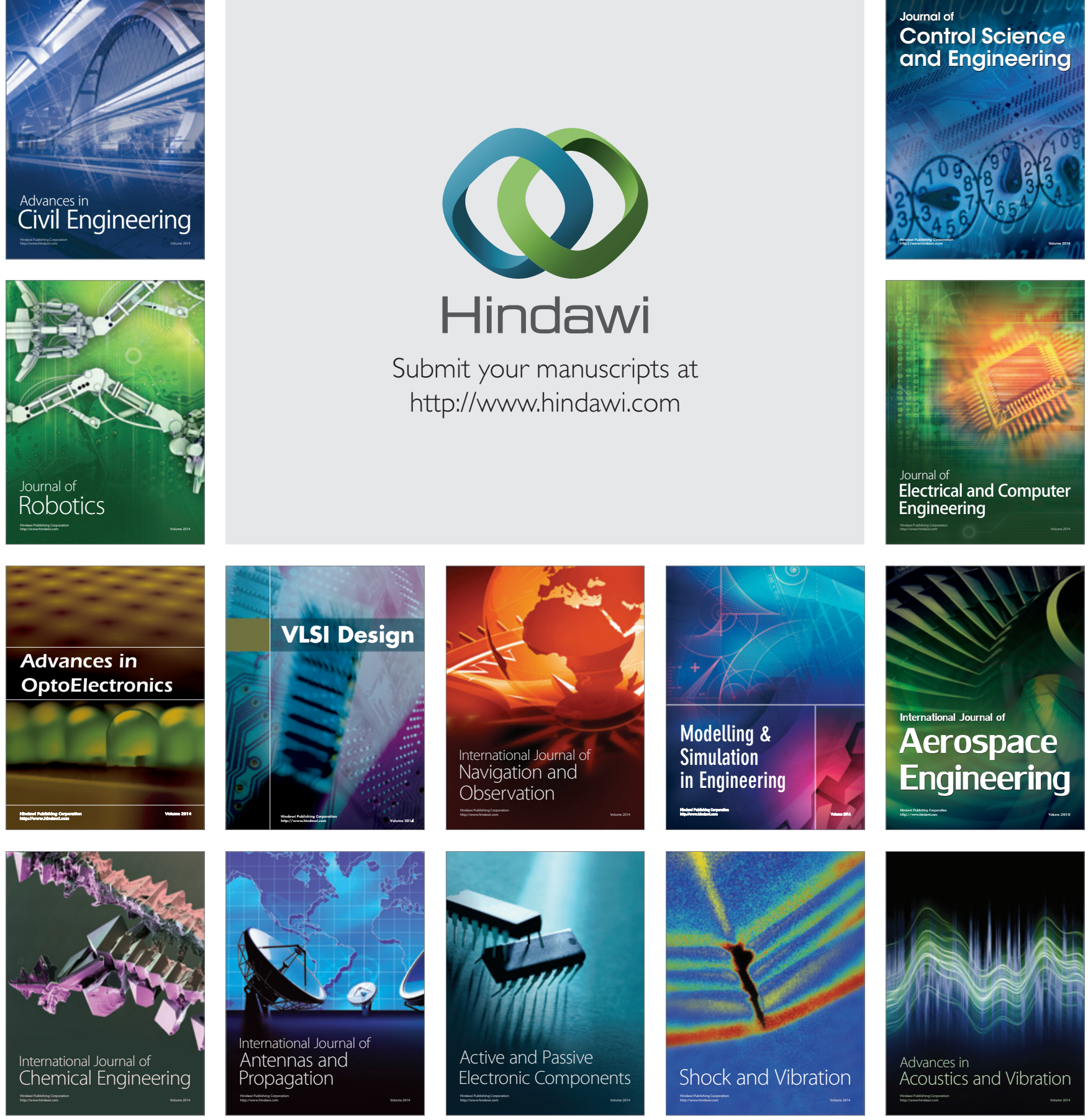